\title{
Etiological Role of Sulfate-Reducing Bacteria in the Development of Inflammatory Bowel Diseases and Ulcerative Colitis
}

\author{
Ivan V. Kushkevych ${ }^{*}$ \\ Laboratory of Molecular Biology and Clinical Biochemistry, Institute of Animal Biology of NAAS of Ukraine, Lviv, Ukraine \\ *Corresponding author: ivan.kushkevych@gmail.com
}

Received August 04, 2014; Revised August 15, 2014; Accepted August 29, 2014

\begin{abstract}
Inflammatory bowel disease and ulcerative colitis are complex multifactorial diseases of unknown etiology. The etiological role of the sulfate-reducing bacteria Desulfovibrio piger Vib-7 and Desulfomicrobium sp. Rod-9 in the development of these inflammatory diseases in rats was studied. Applying sulfate-reducing bacteria, ulcerative colitis in the animals was experimentally induced at the fist time. In total 45 animals in three groups were separated and used in this experiment. Animals in the first group received the standard diet containing a special certified feed for rats and were used as a control. The second group received the standard feed and a dose $(1 \mathrm{ml}$ per each day) of modified Kravtsov-Sorokin's liquid medium for the initiation of own potential animal intestinal microflora of sulfate-reducing bacteria. The third group of the animals received the standard feed and dose of a suspension with D. piger Vib-7 and Desulfomicrobium sp. Rod-9 (at a ratio of 1 to 1 ) in the medium. The changes in the colonic microbiota under these conditions were studied. The bacteria belonging to the normal colonic microbiota were associated with the etiology of inflammatory bowel disease and ulcerative colitis. The concentration of sulfide and acetate in feces from different sections of the large intestine of the rats was determined. The level of ulcerations in the second and third groups of sick animals under the specific conditions was demonstrated. The described experimental results could be particularly useful for the study of inflammatory bowel disease and ulcerative colitis and its therapeutic strategy. These data are also indispensable to apply into mechanistic details that will facilitate better preclinical drug/therapy design to target specific components involved in the disease pathogenesis.
\end{abstract}

Keywords: sulfate-reducing bacteria, sulfide, acetate, inflammatory bowel diseases, ulcerative colitis

Cite This Article: Ivan V. Kushkevych, "Etiological Role of Sulfate-Reducing Bacteria in the Development of Inflammatory Bowel Diseases and Ulcerative Colitis." American Journal of Infectious Diseases and Microbiology, vol. 2, no. 3 (2014): 63-73. doi: 10.12691/ajidm-2-3-5.

\section{Introduction}

Sulfate-reducing bacteria Desulfovibrio, Desulfomicrobium, Desulfobulbus, Desulfobacter, Desulfomonas and Desulfotomaculum genera belong to the intestinal microbiota of humans and animals [1,2,3]. They consume sulfate as an electron acceptor and organic compounds as an electron donor and carbon source in the process of dissimilatory sulfate reduction $[4,5,6]$. Lactate is the most common substrate used by the species belonging to the intestinal sulfate-reducing bacteria [7,8]. The species of Desulfovibrio and Desulfomicrobium genera oxidize lactate incompletely to acetate [1,5,9]. Lactate oxidation to acetate occurs together with the concurrent reduction of sulfate to sulfide $[4,6,10,11]$. The presence of lactate and sulfate in the human intestine contributes to the intensive bacteria growth and the accumulation of their final metabolic products, acetate and hydrogen sulfide, which are toxic, mutagenic and cancerogenic to epithelial intestinal cells $[2,7,8,13,14]$.
The increased number of sulfate-reducing bacteria and intense process of dissimilatory sulfate reduction in the gut can cause inflammatory bowel diseases of humans and animals $[8,12,15,16]$. These bacteria are often found in persons with rheumatic diseases, and with ankylosing spondylitis, etc. $[1,18,19]$. There is also an assumption that sulfate-reducing bacteria can cause some forms of cancer of the rectum through the formation of hydrogen sulfide [14,15,20], which affects the metabolism of intestinal cells and give rise to various inflammatory bowel diseases $[12,13,17,21]$. The species of Desulfovibrio genus cause bloody diarrhea, weight loss, anorexia, epithelial hyperplasia, abscesses and inflammatory infiltrates in animals and humans [2,3,8,14,15,19]. The increased number of sulfate-reducing bacteria was found in feces from people with ulcerative colitis compared with healthy individuals $[2,3,10,17,19]$. The injection of these bacteria in hamster intestine caused infection is clinically similar to human colitis [14,15]. Animal models of inflammatory bowel disease and ulcerative colitis have been used for over fifty years [21,22,23,24]. 
Subsequent refinement and development of this model led to a variety of chemically-induced models [21]. These models have recently been comprehensively reviewed in the context of the role of intestinal bacteria in the development of inflammatory bowel disease [21,25].

Animal models of colitis which can cause disease experimentally in the presence of sulfate containing compounds are increasingly used by the scientists. Additions of carrageenan, sodium lignosulfonate and amylopectin sulfate in drinking water of guinea pigs and rabbits caused damage to the intestine which had clinically symptoms similar to human ulcerative colitis [14]. The inflammation was localized in distal to the cecum. In humans, the disease always occurs in the distal colon. The severity of the disease was correlated with sulfate content in the polymer. The mechanisms of these diseases are not fully understood. However, the activation of the immune response occurred in these animals after the introduction of sulfates in food, and damage to the intestinal mucosa was also observed [14,15,16]. The presence of carrageenan in the intestine of mice as well as its interaction with normal microbiota can also cause colitis. Chronic colitis in mice and hamsters using dextran sulfate was experimental induced [8,22].

The studies of animal models of intestinal inflammation and ulcerative colitis in humans showed that intestinal bacteria play an important role in the initiation and maintenance of the inflammation [18,22]. The presence of sulfate is also important in the development of these diseases $[1,2,8,9,12,17]$. The disruption of the qualitative and quantitative composition of the microflora of the digestive tract may be due to infections, stress, antibiotics, chemotherapy, radiation action, poisoning, poor nutrition, and others $[8,12]$. The changes in the composition of intestinal microflora, in turn, can cause disorders of metabolism processes and immune status, which indicates the close relationships between host and his normal microflora [18].

In literature, there are a lot of data on the role of various microorganisms in the development of inflammatory bowel diseases and ulcerative colitis as well as the characteristics of the animal models of these intestinal inflammations which were chemically created $[2,3,7,12,13,16,17,19,24]$. However, the role of intestinal sulfate-reducing bacteria Desulfovibrio piger and Desulfomicrobium sp. in the development of these diseases has never been well-characterized. As far as it is aware, the creation of the inflammatory bowel diseases and ulcerative colitis using D. piger and Desulfomicrobium sp. has not been reported yet.

The aim of this work was to study the etiological role of the intestinal sulfate-reducing bacteria Desulfovibrio piger Vib-7 and Desulfomicrobium sp. Rod-9 in the inflammatory bowel diseases as well as to create of ulcerative colitis in rats by applying these bacterial strains.

\section{Materials and Methods}

Animals. All manipulations with the animals were carried out under the principles of the "European Convention for the Protection of Vertebrate Animals Used for Experimental and Other Scientific Purposes" (Strasbourg, 1986), the Decision of the First National Congress on
Bioethics (Kyiv, 2001) and the Law of Ukraine "On Protection Animals from Brutal Treatment” (Kyiv, 2006).

The laboratory male rats (seven months age, weight $250 \pm 25$ g) were held under standard vivarium conditions. The laboratory animals belonged to fourth class of cleanliness by microbiological status. The diet contained a standard certified feed PK-120-1 for laboratory rats.

In total 45 animals in three groups were separated (15 animals in each group) and used in this experiment. The first group received the standard diet containing the certified feed and was used as a control; second and third groups were taken for creation of an experimental animal model of ulcerative colitis. Animals of the second group received the standard feed and dose ( $1 \mathrm{ml}$ per each day) of modified Kravtsov-Sorokin's liquid medium (without Mohr's salt) [26] for the initiation of their own potential microflora of the sulfate-reducing bacteria. Third group animals received the standard feed and dose of a suspension with $D$. piger Vib-7 and Desulfomicrobium sp. Rod-9 (at a ratio of 1 to 1 ) in the medium ( $1 \mathrm{ml}$ per each day; the concentration of bacterial cells was $3 \mathrm{mg} / \mathrm{ml}$ ). The strains of the sulfate-reducing bacteria were previously isolated from the human large intestine and identified by sequence analysis of the 16S rRNA gene [26,27]. Kravtsov-Sorokin's liquid medium or suspension with microorganisms was peroral introduced into the stomach using a metal probe directly during 25 days.

The qualitative and quantitative composition of intestinal microflora was studied in the animals of the control and experimental groups after 5, 10, 15, 20, 25 days from the beginning of injection of the medium or bacterial cell suspension. The animals were decapitated under ether anesthesia and the isolated colonic segments were selected for these studies.

Bacteriology. To study of the lumen microflora of the rat colon, squeezed contents of 3-cm size segment of the distal colon was taken by sterile forceps under sterile conditions and weighed. The weighed sample was added in a sterile tube with tenfold volume of sterile isotonic sodium chloride solution (dilution $10^{-1}$ ). The mixture was thoroughly triturated with a sterile glass rod to form a homogeneous mass. Subsequently, a series of ten-fold dilutions (from $10^{-2} \ldots 10^{-12}$ ) in sterile isotonic sodium chloride solution was prepared from the homogenate. After ten-fold serial dilutions in sterile isotonic sodium chloride solution, the dimensional volumes $(0.1 \mathrm{ml})$ were passaged on the selective microbial culture media for each genus of Enterobacteriacea family. Identification of the isolated cultures of anaerobic and aerobic microorganisms was carried out by morphological, tinctirial, cultural, biochemical properties [5] using test system API 20E for identification of Enterobacteriacea family. The sulfatereducing bacteria were grown in a nutrition-modified Kravtsov-Sorokin's liquid medium and their identification was carried out as described previously [26]. Growth of the sulfate-reducing bacteria was evidenced by black staining (ferrous sulfide) of the media. After incubation, the number of colonies was counted and population level of each group of microorganisms in $\log _{10}$ colony-forming units (CFU) per gram wet weight stool was recorded and used in calculations.

The colon microbiota was assessed by Sustainability Index $(S I, \%)$ and detection frequencies $\left(P_{i}\right)$ : 


$$
S I=p / P \times 100 ; P_{i}=A / B,
$$

where $S I$ is sustainability index (\%), $p$ is number of samples containing the studied bacterial strain, $P$ is total number of samples containing all isolates of the bacteria; $A$ is the number of strains of the specific type, $B$ is total number of all strains.

Sulfide release by incubated fecal specimens. After 5, $10,15,20,25$ days from the beginning of injection of the medium and bacterial cells suspension, fresh fecal samples from different sections of the large intestine were passed into preweighed containers: 20 -ml polypropylene syringes for measurement of $\mathrm{H}_{2} \mathrm{~S}$ release; pre-weighed 50-ml test tubes containing $5 \mathrm{ml}$ of $2 \%$ zinc acetate (ice cold) for $\mathrm{H}_{2} \mathrm{~S}$ and acetate concentration measurements; and 4-ml vials for sulfate-reducing bacteria culture [28].

Following collection of the fecal sample, the plunger was immediately reinserted into the syringe, the gas space was flushed with nitrogen $\left(\mathrm{N}_{2}\right), 20 \mathrm{ml}$ of $\mathrm{N}_{2}$ was added, and the syringe was sealed with a stopcock. The syringes were incubated at $+37^{\circ} \mathrm{C}$, and at $1,2,4$, and $24 \mathrm{~h}$ the gas volume was recorded and a $0.3-\mathrm{ml}$ gas sample was removed for analysis for $\mathrm{H}_{2} \mathrm{~S}$. At $24 \mathrm{~h}$, the syringes were weighed, and fecal weight determined by difference. The rate of release of $\mathrm{H}_{2} \mathrm{~S}$ per gram wet weight was calculated from the concentration of $\mathrm{H}_{2} \mathrm{~S}$, the volume of the gas space, and fecal weight [28,29].

Sulfide concentration of feces. The vial containing the fecal sample and zinc acetate was weighed and fecal weight determined by difference. The volume of zinc acetate was adjusted to give a 1:100 ratio of feces to zinc acetate and the sample was homogenized. A 0.5-ml aliquot of the homogenate was added to a 20-ml polypropylene syringe, $0.5 \mathrm{ml}$ of $12 \mathrm{~N} \mathrm{HCl}$ was instilled into the syringe, and the syringe was sealed with a stopcock. After $30 \mathrm{~min}$, a $0.3-\mathrm{ml}$ aliquot of gas space was analyzed for $\mathrm{H}_{2} \mathrm{~S}[28,29]$.

Acetate concentration of feces. The fresh fecal samples were also used for measurements of acetate concentration in different sections of the large intestine after $5,10,15$, 20, 25 days. The concentration of acetate was determined using Acetate Colorimetric Assay Kit (Sigma-Aldrich, Catalog Number MAK086).

Statistical analysis. Statistical calculations of the results were carried out using the software MS Office and Origin computer programs. All data were expressed as mean R standard error of the mean (S.E.M.). The research results were treated by the methods of variation statistics using Student $t$-test. Characteristics of different treatment groups were compared with the unpaired t-test. Paired ttests were also used to examine changes over the trial period within each group. The equation of the straight line that the best approximates the experimental data was calculated by the method of least squares. The absolute value of the correlation coefficient $r$ was from 0.90 to 0.98 . The statistical significance of the calculated parameters of line was tested by the Fisher's F-test. The accurate approximation was when $\mathrm{P} \leq 0.05$ [30].

\section{Results and Discussion}

Microbiological analysis of the intestinal microflora of the rats during the twenty-five days was carried out.
The samples were selected from each group of animals on the fifth, tenth, fifteenth, twentieth, and twenty-fifth day (Figure 1). The results of these studies showed that microflora of the first group of the animals (control) receiving the standard diet during twenty-five days was almost unchanged. In total, fifteen genera of the bacteria were isolated, in particular Bacteroides, Prevotella, Bifidobacterium, Lactobacillus, Eubacterium, Fusobacterium, Clostridium, Peptococcus, Peptostrepto-coccus, E. coli (lactose-positive), E. coli (lactose-negative), Proteus, Klebsiella, Enterococcus, Staphylococcus, Streptococcus, Candida, and some sulfate-reducing bacteria. The largest number and predominant of the microorganisms among these genera ( $\left.\log _{10} \mathrm{CFU} / \mathrm{gram}\right)$ : Bacteroides (9.14 \pm 0.89 ), Prevotella (9.11 \pm 0.87$), \quad$ Lactobacillus (9.24 \pm 0.91$)$, Peptococcus (9.08 \pm 0.93$)$, and Bifidobacterium (8.99 \pm 0.86$)$ on the fifth day were detected. A slightly lower number of the intestinal bacterial genera Eubacterium (8.72 \pm 0.85$)$, Fusobacterium (8.25 \pm 0.82$), \quad$ Peptostreptococcus (8.82 \pm 0.78$)$, Entero-coccus (7.84 \pm 0.74$)$, Streptococcus $(6.22 \pm 0.63)$ were observed. The lowest number of the microorganisms E. coli (lactose-positive) (5.61 \pm 0.54$)$, Staphylococcus (5.03 \pm 0.47$)$, Candida (4.42 \pm 0.39$)$, Proteus (3.24 \pm 0.33$)$, Clostridium (2.72 \pm 0.24$)$, E. coli

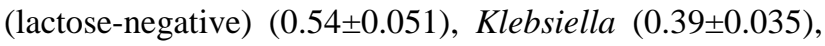
and sulfate-reducing bacteria $(0.58 \pm 0.055)$ were isolated. The quantitative and qualitative ratio of these microorganisms was almost constant during 25 days in the first (control) group of the experimental animals.

Addition a dose of the modified Kravtsov-Sorokin's liquid medium which contained sulfate ions ( $1 \mathrm{ml}$ per each day) in the standard animal diet (second group) and the dose of a suspension with $D$. piger Vib-7 and Desulfomicrobium sp. Rod-9 (at a ratio of 1 to 1) in the medium (third group), the changes in the microflora were already observed on the fifth day of the experiment. In this case, the injection of the medium to animal gastrointestinal tract caused a reduction in the number of Lactobacillus (20\%), Peptococcus (14\%), Candida (21\%), and an increase in the number of E. coli (lactose-negative) (70\%), Proteus (13\%), Klebsiella and Staphylococcus (28\%), and the sulfate-reducing bacteria (86\%) in the second animal group on the fifth day. The injection of the dose of the suspension with the strains of sulfate-reducing bacteria to the tract led to the reduction in the number of Lactobacillus (30\%), Peptococcus (22\%), Candida (25\%), and the increase in the number of Clostridium (40\%), E. coli (lactose-negative) (78\%), Proteus (26\%), Klebsiella (36\%) and Staphylococcus (30\%), and the sulfatereducing bacteria (92\%) in third animal group on the fifth day of the detection compared to the control.

As this study shows, the qualitative and quantitative changes in the intestinal microflora depended on the time of the introduction of the dose of the sulfate containing medium or bacterial suspension. Each additional dose of the medium or the suspension caused significant violation of the bowel microbiocenosis in the rats on the $10^{\text {th }}, 15^{\text {th }}$, $20^{\text {th }}, 25^{\text {th }}$ day of detection.

The greatest qualitative and quantitative changes in the microflora in the second and third animal groups were observed on the $25^{\text {th }}$ day. Under these conditions, everyday introduction of the dose of the medium or the suspension to animal gastrointestinal tract led to significant reduction in the number of Bifidobacterium and 
Lactobacillus (90\%), Eubacterium (30\%), Peptococ-cus (73\%), Peptostreptococcus (57\%), Enterococcus (86\%), Candida (77\%), and the increase in the number of Clostridium (71\%), E. coli (lactose-positive) (79\%), E. coli (lactose-negative) (94\%), Proteus (63\%), Klebsiella (80\%), Staphylococcus (43\%), and the sulfate-reducing bacteria (95\%) in the second animal group compared to the control on the $25^{\text {th }}$ day.
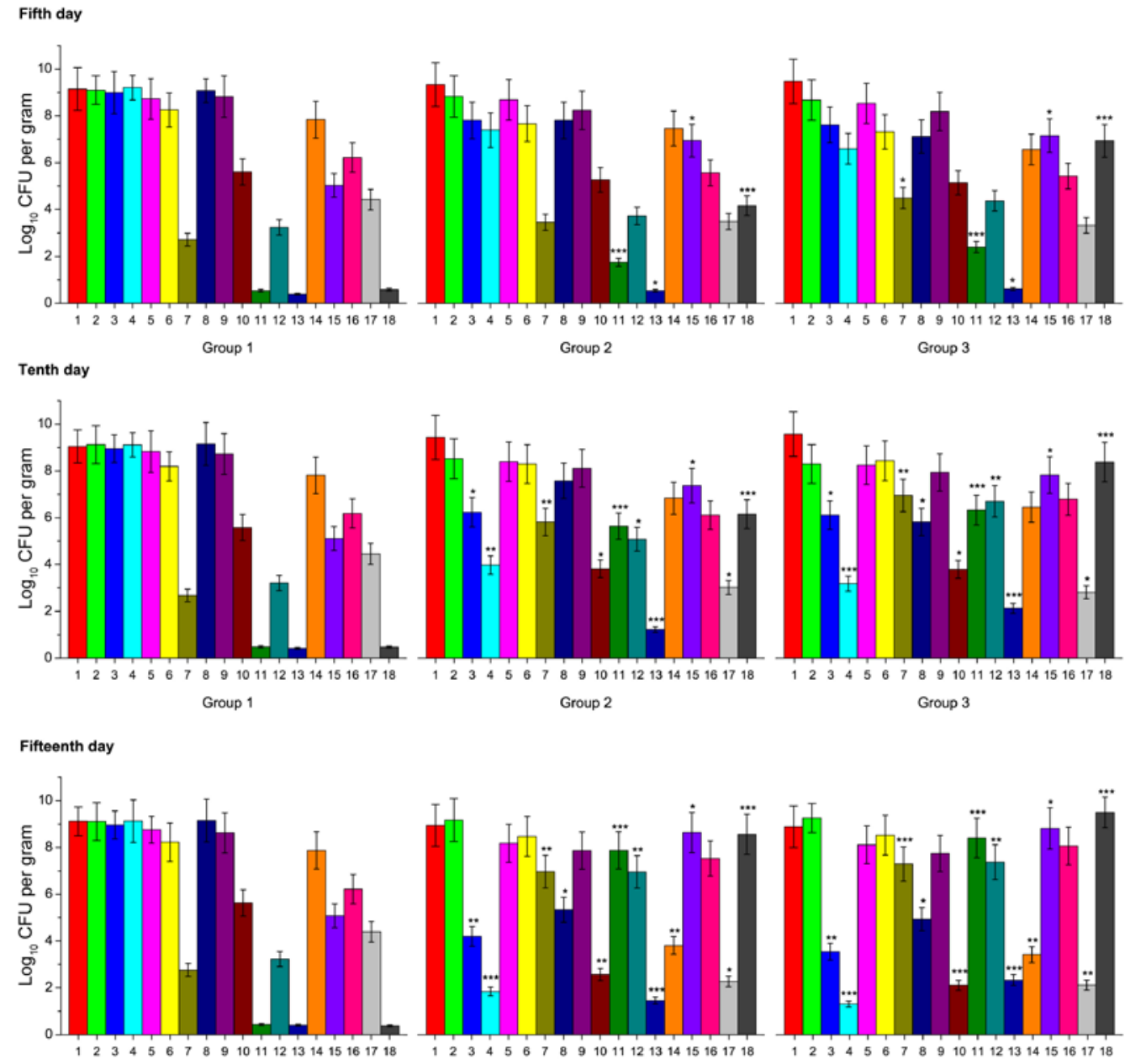

Group 1
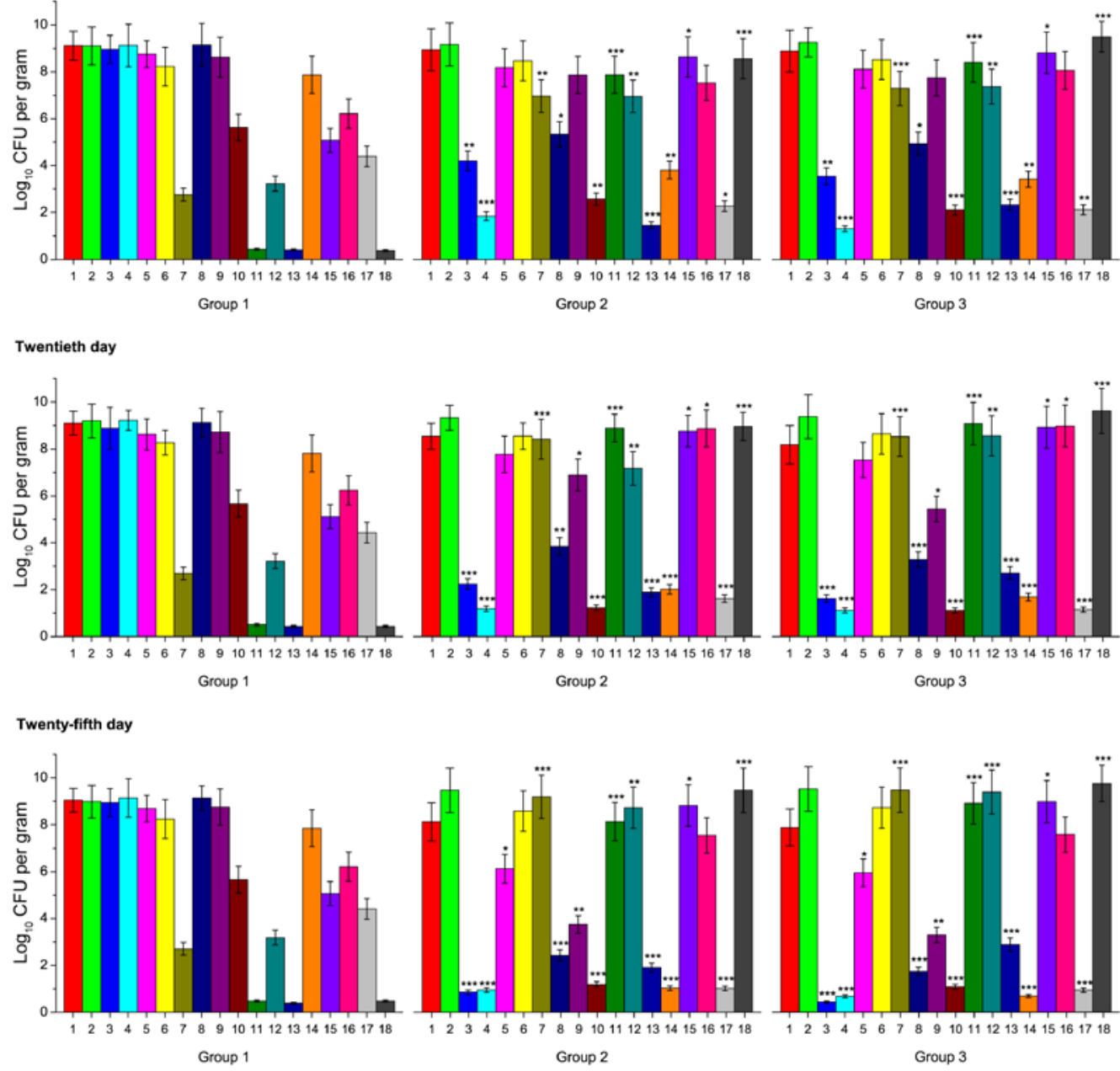

Figure 1. The microflora of the distal colon in the rats under the effect of specific conditions during twenty-five days: 1 - Bacteroides, 2 - Prevotella, 3 - Bifidobacterium, 4 - Lactobacillus, 5 - Eubacterium, 6 - Fusobacterium, 7 - Clostridium, 8 - Peptococcus, 9 - Peptostreptococcus, 10 - Escherichia coli (lactose-positive), 11 - Escherichia coli (lactose-negative), 12 - Proteus, 13 - Klebsiella, 14 - Enterococcus, 15 - Staphylococcus, 16 Streptococcus, 17 - Candida, 18 - Sulfate-reducing bacteria. Statistical significance of the values $\mathrm{M} \pm \mathrm{m}, \mathrm{n}=3 ;{ }^{*} \mathrm{P}<0.05,{ }^{* *} \mathrm{P}<0.01,{ }^{* * *} \mathrm{P}<0.001$, compared to first (control) animals group 
Significant reduction in the number of Lactobacillus and Bifidobacterium (93-95\%), Eubacterium (32\%), Pepto-coccus (81\%), Peptostreptococcus (62\%), Enterococcus (91\%), Candida (79\%), and the increase in the number of Clostridium (73\%), E. coli (lactose-positive) (81\%), E. coli (lactose-negative) (96\%), Proteus (67\%), Klebsiella (86\%), Staphylococcus (45\%), and the sulfatereducing bacteria (97\%) compared to the control in the third animal group was observed on the $25^{\text {th }}$ day.
The sustainability index (SI, \%) and frequencies $\left(P_{i}\right)$ of the microflora is very important indicator of the intestinal bacteria number to characterize the bowel conditions and its microbiota.

The analysis of the sustainability index and frequencies of the intestinal microflora of different animal group on the twenty-fifth day was carried out. The results of this study showed that the $S I$ and $P_{i}$ data were significantly different for the three groups of animals (Table 1).

Table 1. Sustainability index and frequencies of the microflora of the distal colon in the rats

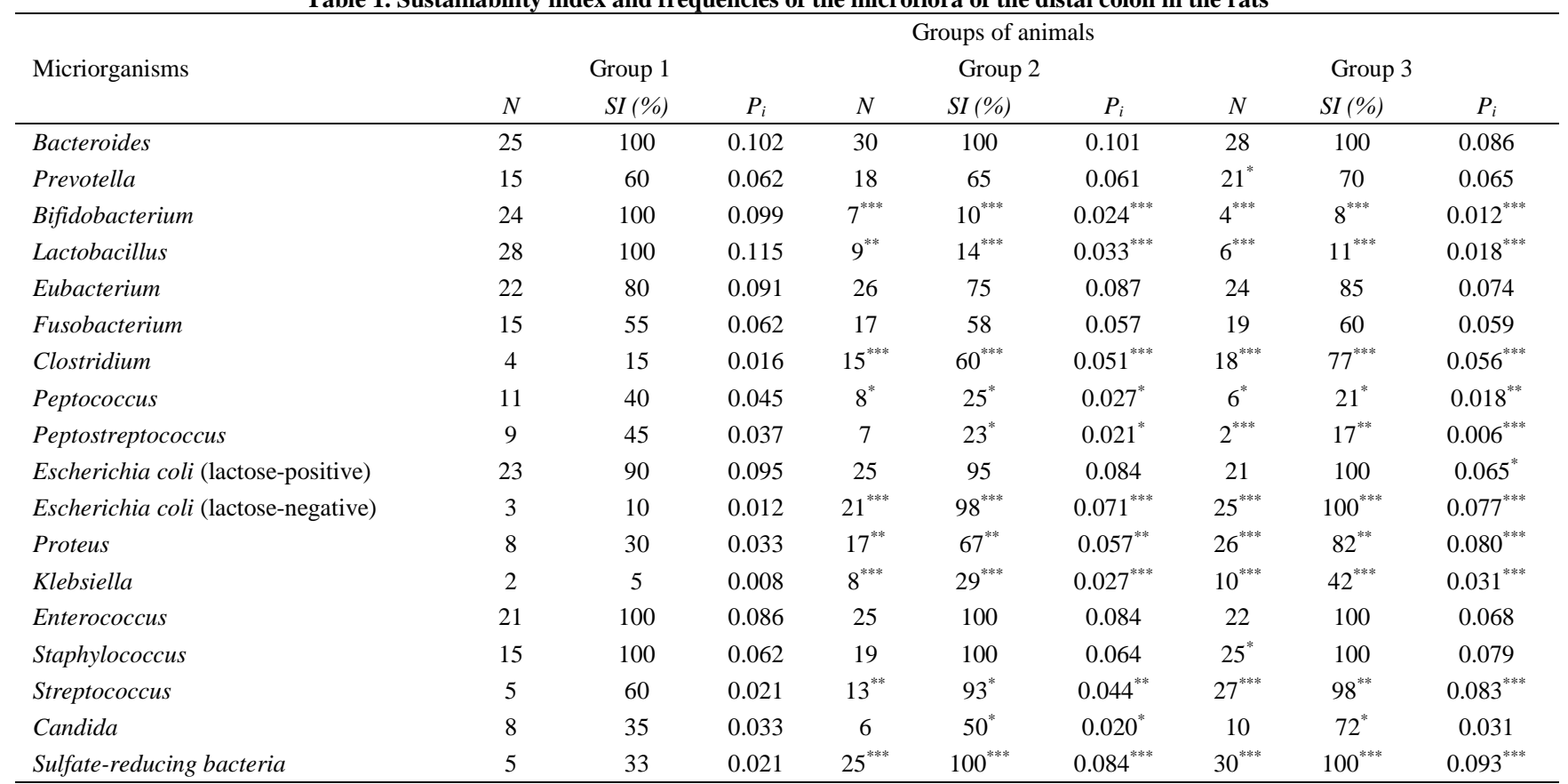

Comment: $N$ is number of the isolated strains; $S I$ is sustainability index; $P_{i}$ is frequencies. Data were calculated for intestinal microflora on twenty-fifth day. Statistical significance of the values $\mathrm{M} \pm \mathrm{m}, \mathrm{n}=3 ;{ }^{*} \mathrm{P}<0.05,{ }^{* * *} \mathrm{P}<0.01,{ }^{* * *} \mathrm{P}<0.001$, compared to first (control) animals group.

These data were also consistent to previously obtained results of the qualitative and quantitative changes in the microflora in the rats on the twenty-fifth day. The predominant bacteria of the colon of the first (control) animals group were Bacteroides $\left(N_{25} ; S I_{100 \%} ; P_{i} 0.102\right)$, Prevotella $\left(N_{15} ; S I_{60 \%} ; P_{i 0.062}\right)$, Bifidobacterium $\left(N_{24} ; S I_{100 \%}\right.$; $P_{i}$ 0.099), Lactobacillus $\left(N_{28} ; S I_{100 \%} ; P_{i} 0.115\right)$, Eubacterium $\left(N_{22} ; S I_{80 \%} ; P_{i 0.091}\right)$, Fusobacterium $\left(N_{15} ; S I_{55 \%} ; P_{i 0.062}\right)$, Escherichia coli (lactose-positive) ( $\left.N_{23} ; S I_{90 \%} ; P_{i} 0_{0.095}\right)$, Enterococcus $\left(N_{21} ; S I_{100 \%} ; P_{i 0.086}\right)$. These data indicated that the bacterial genera were dominant representatives of the obligate microflora of the lumen distal colon in the rats. It should be also noted that the values $S I$ and $P_{i}$ for genera Peptostreptococcus $\left(N_{9} ; S I_{45 \%} ; P_{i 0.037}\right)$, Peptococcus $\left(N_{11}\right.$; $\left.S I_{40 \%} ; P_{i 0.045}\right)$, and for yeast Candida genus $\left(N_{8} ; S I_{35 \%} ; P_{i}\right.$ 0.033) were lower compared to other isolated genera. The sustainability index and the frequency of detection were also lower for Proteus $\left(N_{8} ; S I_{30 \%} ; P_{i 0.033}\right)$, Klebsiella $\left(N_{2}\right.$; $\left.S I_{5 \%} ; P_{i} 0.008\right)$, Streptococcus $\left(N_{5} ; S I_{60 \%} ; P_{i} 0.021\right)$ and Clostridium ( $N_{4} ; S I_{15 \%} ; P_{i}$ 0.016) genera, and sulfatereducing bacteria $\left(N_{5} ; S I_{33 \%} ; P_{i} 0.021\right)$. Thus, these microorganisms were representatives of the facultative, transient microflora of the large intestine of the rats.

Significant changes in the qualitative and quantitative microflora composition of the colon in the second and third animals groups was observed. The most noticeable changes were specific to genera Bifidobacterium $\left(N_{7}\right.$; $S I_{10 \%} ; \quad P_{i}$ 0.024), Lactobacillus $\left(N_{9} ; \quad S I_{14 \%} ; P_{i} \quad 0.033\right)$, Clostridium $\left(N_{15} ; S I_{60 \%} ; P_{i 0.051}\right)$, Peptococcus $\left(N_{8} ; S I_{25 \%} ; P_{i}\right.$ 0.027), Peptostreptococcus $\left(N_{7} ; S I_{23 \%} ; P_{i} 0.021\right)$, E. coli (lactose-negative) $\left(N_{21} ; S I_{98 \%} ; P_{i 0.071}\right)$, Proteus $\left(N_{17} ; S I_{67 \%}\right.$; $\left.P_{i 0.057}\right)$, Klebsiella $\left(N_{8} ; S I_{29 \%} ; P_{i 0.027}\right)$, Staphylococcus $\left(N_{19}\right.$; $\left.S I_{100 \%} ; P_{i 0.064}\right)$, Streptococcus $\left(N_{13} ; S I_{93 \%} ; P_{i} 0.044\right)$, and sulfate-reducing bacteria $\left(N_{25} ; S I_{100 \%} ; P_{i 0.084}\right)$ for animals of the second group. Similar results of the sustainability index and frequencies of the microflora of the third animals group were obtained for the following genera Bifidobacterium $\left(N_{4} ; S I_{8 \%} ; P_{i} 0.012\right)$, Lactobacillus $\left(N_{6}\right.$; $S I_{11 \%} ; \quad P_{i}$ 0.018), Clostridium $\left(N_{18} ; \quad S I_{77 \%} ; P_{i}{ }_{0.056}\right)$, Peptococcus $\left(N_{6} ; S_{21 \%} ; P_{i 0.018}\right)$, Peptostreptococcus $\left(N_{2}\right.$; $S I_{17 \%} ; P_{i}$ 0.006), E. coli (lactose-negative) $\left(N_{25} ; S I_{100 \%}\right.$; $\left.P_{i 0.077}\right)$, Proteus $\left(N_{26} ; S I_{82 \%} ; P_{i 0.080}\right)$, Klebsiella $\left(N_{10} ; S I_{42 \%}\right.$; $P_{i}$ 0.031), Staphylococcus $\left(N_{25} ; S I_{100 \%} ; P_{i} 0.079\right)$, Streptococcus $\left(N_{27} ; S I_{98 \%} ; P_{i} 0.083\right)$, and the sulfate-reducing bacteria $\left(N_{30} ; S I_{100 \%} ; P_{i} 0.093\right)$. Thus, the most significant changes were characteristic for the following genera Bifidobacterium, Lactobacillus, Clostridium, Peptococcus, Peptostreptococcus, E. coli (lactose-positive), E. coli (lactose-negative), Proteus, Klebsiella, Staphylococcus, Streptococcus, and the sulfate-reducing bacteria.

Colonic bacteria produce large quantities of hydrogen sulfide $[1,4,8,11]$. It is known that sulfide is produced by a group of bacteria, sulfate-reducing bacteria that obtain energy via reactions involving the reduction of sulfate to sulfide $[2,3,14,15]$. In vitro studies indicate that fecal bacteria liberate sulfide far more efficiently from organic sulfur-containing compounds than from sulfate [11,28].

As already mentioned above, the sulfate-reducing bacteria Desulfovibrio and Desulfomicrobium genera oxidize organic compounds incompletely to acetate $[5,6,8]$. 
The oxidation to acetate occurs together with the

concurrent reduction of sulfate to sulfide $[1,4,5,8]$.
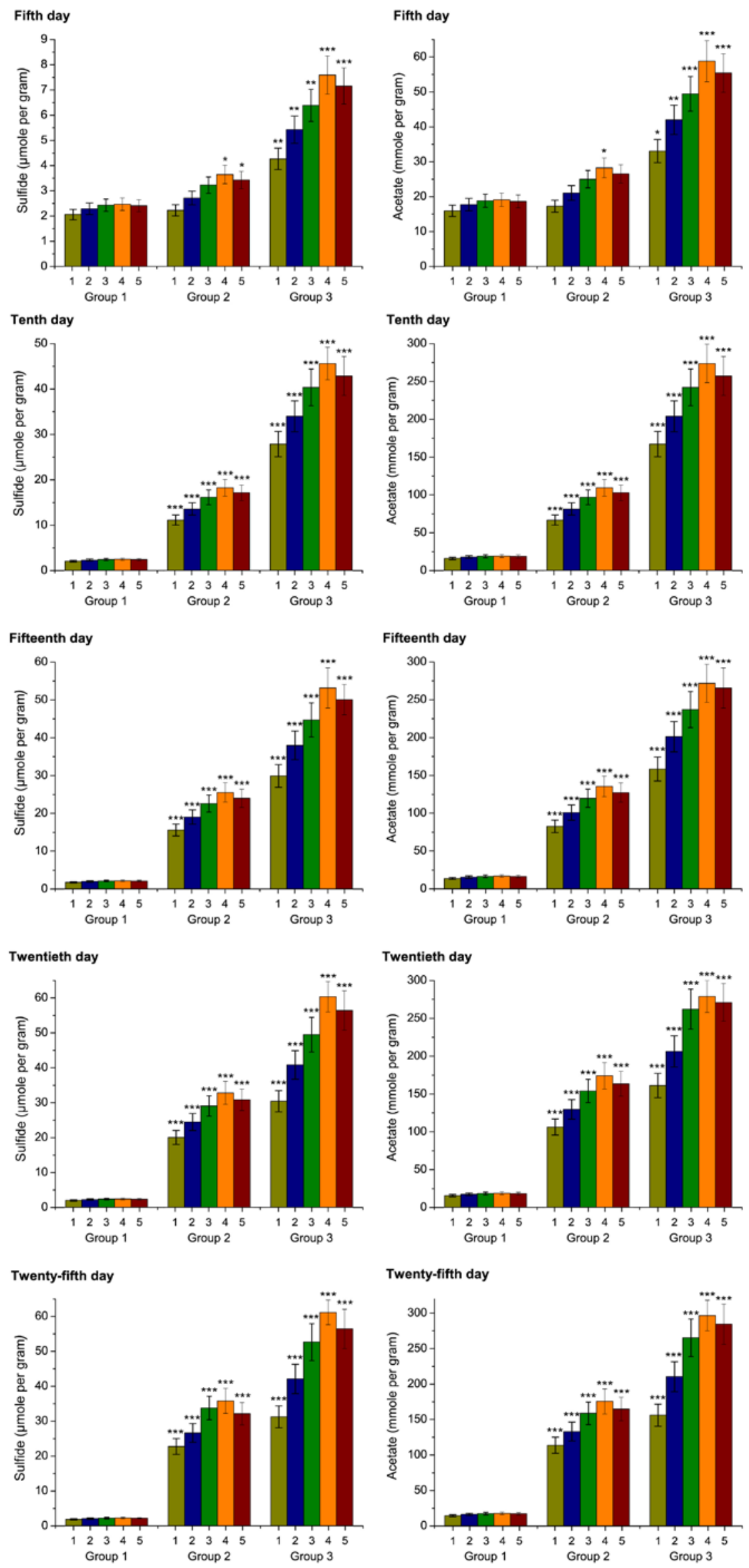

Figure 2. Sulfide and acetate concentration in fecal samples from different sections of the large intestine of the rats: 1 - Caecum, 2 - Ascending colon, 3 - Transverse colon, 4 - Descending colon, 5 - Rectum. Statistical significance of the values $\mathrm{M} \pm \mathrm{m}, \mathrm{n}=3 ;{ }^{*} \mathrm{P}<0.05,{ }^{* * *} \mathrm{P}<0.01,{ }^{* * * *} \mathrm{P}<0.001$, compared to first animals group (control) 
Sulfide and acetate concentration in the fecal samples from different sections of the large intestine of the rats, in particular caecum, ascending colon, transverse colon, descending colon, and rectum was determined (Figure 2). The results of these studies showed that the level of sulfide and acetate in feces in the first group (control) during the 25 days was almost unchanged.

The concentration of sulfide and acetate in fecal samples from different sections of the large intestine was slightly different from each other. The values of concentration (in $\mu$ mole per gram and mmole per gram, respectively) in the fecal samples from caecum $(2.08 \pm 0.19$ and 16.10 \pm 1.53$)$, ascending colon (2.31 \pm 0.21 and $17.88 \pm 1.74)$, transverse colon ( $2.45 \pm 0.23$ and $18.96 \pm 1.81)$, descending colon (2.49 \pm 0.22 and $19.27 \pm 1.88)$, and rectum (2.43 \pm 0.24 and $18.81 \pm 1.79)$ was determined on the fifth day for sulfide and acetate, respectively. The quantitative ratio of these compounds was almost constant during twenty-five days in the first group of the experimental animals.

Significant changes in the concentration of sulfide and acetate in all sections of the large intestine in the second and third group of the rats on the fifth day of the experiment were already observed. As showed, the highest level of sulfide and acetate was accumulated in the descending colon of the second and third animals during
25 days. In this case, sulfide and acetate concentration increased directly proportional to the duration of introduction of the dose of the medium or the suspension with the sulfate-reducing bacteria. The values of sulfide and acetate concentration in the fecal samples from descending colon were increased respectively for the second and third group of the rats by 32 and $65 \%$ as well as 35 and $69 \%$ compared to the control on the fifth day.

More significant quantitative changes in concentration of these compounds and their substantial increase were observed on the twenty fifth day. Under these conditions, the level of sulfide and acetate in the descending colon increased respectively by 88 and $95 \%$ for the second group as well as 93 and $97 \%$ for the third group of the animals.

A correlation analysis is related in the sense that both deal with relationships among variables. The correlation coefficient is a measure of linear association between two variables [31]. The correlation analysis between parameters of sulfide and acetate accumulation in descending colon in the animal groups during 25 days was carried out.

The correlation coefficients $(r)$ between these parameters were defined (Table 2). A strong inversely positive correlation between sulfide and acetate accumulation was demonstrated.

Table 2. Correlation coefficients $(r)$ between parameters of sulfide and acetate accumulation

\begin{tabular}{|c|c|c|c|c|}
\hline \multirow{2}{*}{\multicolumn{2}{|c|}{ Day of detection }} & \multicolumn{3}{|c|}{ Sulfide } \\
\hline & & Group 1 & Group 2 & Group 3 \\
\hline \multirow{5}{*}{ 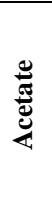 } & Fifth & $0.979 \pm 0.095$ & $0.974 \pm 0.095$ & $0.976 \pm 0.095$ \\
\hline & Tenth & $0.965 \pm 0.094$ & $0.958 \pm 0.093$ & $0.964 \pm 0.094$ \\
\hline & Fifteenth & $0.951 \pm 0.092$ & $0.969 \pm 0.094$ & $0.975 \pm 0.095$ \\
\hline & Twentieth & $0.966 \pm 0.094$ & $0.978 \pm 0.095$ & $0.953 \pm 0.093$ \\
\hline & Twenty-fifth & $0.977 \pm 0.095$ & $0.962 \pm 0.093$ & $0.958 \pm 0.093$ \\
\hline
\end{tabular}

Comments: Correlation coefficients between parameters of accumulation of sulfide and acetate were calculated based on data obtained from feces of all colon sections. Statistical significance of the values $\mathrm{M} \pm \mathrm{m}, \mathrm{n}=3$.

The correlation coefficient ranges from -1.0 to +1.0 . The closer $r$ is to +1 or -1 , the more closely the two variables are related. If $\mathrm{r}$ is close to 0 , it means there is no relationship between the variables. If $r$ is positive, it means that as one variable gets larger the other gets larger. If $\mathrm{r}$ is negative it means that as one gets larger, the other gets smaller (often called an "inverse" correlation). While correlation coefficients are normally reported as $r=$ (a value between -1 and +1 ), squaring them makes then

easier to understand. Values between 0.7 and 1.0 (-0.7 and -1.0) indicate a strong positive (negative) linear relationship via a firm linear rule [30,31].

The systematic statistical analysis of the parameters of sulfide and acetate accumulation was also carried out. The results of the studies showed that the variance, pooled variance, t-statistics in the experimental groups of animals were quite variable on the 25 day of the detection (Table 3).

Table 3. The systematic statistical analysis of the parameters of sulfide and acetate accumulation

\begin{tabular}{cccccc}
\hline \multirow{2}{*}{ Parameters } & \multicolumn{2}{c}{ Group 1} & \multicolumn{2}{c}{ Group 2 } & Group 3 \\
& Sulfide & Acerate & Sulfide & Acerate & Sulfide \\
\hline Average & $2.15 \pm 0.20$ & $16.66 \pm 1.62$ & $30 \pm 2.93^{* * *}$ & $149 \pm 14.49^{* * *}$ & $49 \pm 4.74^{* * *}$ \\
Variance & $0.028 \pm 0.003$ & $1.655 \pm 0.161$ & $29 \pm 2.81^{* * *}$ & $636 \pm 61^{* * *}$ & $146 \pm 13^{* * *}$ \\
Pooled Variance & \multicolumn{2}{c}{$0.841 \pm 0.082$} & & $332 \pm 32^{* * *}$ & $246 \pm 23.92^{* * *}$ \\
t-statistics & \multicolumn{2}{c}{$-25 \pm 2.43$} & $-10 \pm 0.98^{* * *}$ & $-7 \pm 0.65^{* * *}$ \\
\hline
\end{tabular}

Comments: The systematic statistical analysis of the parameters of sulfide and acetate accumulation was defined on the twenty-fifth day; observation is equal to 5; t critical one-way is equal to $1.860 \pm 0.181$; $\mathrm{t}$ critical two-way is equal to $2.306 \pm 0.224$. Statistical significance of the values, $\mathrm{M} \pm \mathrm{m}$, $\mathrm{n}=3$; ${ }_{* * *} \mathrm{P}<0.001$, compared to first animals group (control).

These statistical parameters also depended on duration of the experiment. However, $t$ critical one-way (1.860 \pm 0.181$)$ and $t$ critical two-way $(2.306 \pm 0.224)$ were similar for each of the parameters.

Sulfide exists in ionized, non-volatile states ( $\mathrm{S}^{-}$or $\mathrm{HS}^{-}$), as well as volatile $\mathrm{H}_{2} \mathrm{~S}[14,15]$. Levitt et al. have shown that about $95 \%$ of the sulfide produced in the gut is absorbed and the vast majority of the $5 \%$ passed in feces

is tightly bound to other compounds [20,29]. Thus, fecal sulfide concentration appears to reflect sulfide binding capacity rather than intraluminal production [28].

Hydrogen sulfide volatile thiol binds to the heme moiety of cytochrome $a_{3}$, blocking the terminal step in electron transport [32]. When administered systemically, $\mathrm{H}_{2} \mathrm{~S}$ has a median lethal dose $\left(\mathrm{LD}_{50}\right)$ for rodents similar to that of cyanide [28]. The volatility of $\mathrm{H}_{2} \mathrm{~S}$ results in its 
rapid dissociation from fecal material, and this gas has very high tissue permeability [33].

Thus, the colonocyte is exposed to virtually the entire bacterial output of this compound, in contrast to other potentially toxic bacterial metabolites, which remain in feces or which have low permeability for the colonic mucosa [28]. While the colonocyte efficiently detoxifies $\mathrm{H}_{2} \mathrm{~S}$ via conversion to thiosulfate [20,29], there has been speculation that this highly toxic compound could play a pathogenetic role in colonic diseases, particularly ulcerative colitis [34]. Sulfide also has been linked to colon neoplasia via the observation that sulfide exposure induced proliferation in the upper crypt region of the colonic mucosa [35,36,37], a finding associated with mucosal hyperplasia [28]. Hydrogen sulfide is a highly toxic compound that has a median lethal concentration on the same order of magnitude as that of cyanide [38]. The potential for local toxicity of sulfide in the colon has been studied by Roediger et al. [35,36], who incubated human and rat colonic epithelial cells with a range of sulfide concentrations [37]. Low concentrations of sulfide inhibited butyrate oxidation, whereas higher concentrations were required for inhibition of glucose oxidation. Of interest are studies showing that colonic mucosal biopsy specimens from people with moderately severe ulcerative colitis similarly demonstrate inhibition of butyrate oxidation with preservation of glucose metabolism [34,37], whereas both reactions are altered in severe colitis. In animals, inhibition of fatty acid oxidation via rectal instillation of sodium 2-bromo-octanoate produced a colitis resembling ulcerative colitis [15].
Colonic sulfate-reducing bacteria produce hydrogen sulfide and acetate, the toxic compounds postulated to play a pathogenetic role in the inflammatory bowel diseases and ulcerative colitis. Colonic sulfide exposure has previously been assessed via measurements of fecal sulfide concentration [20]. Levitt et al. have shown that $1 \%$ of fecal sulfide of rats was free, the remainder being bound in soluble and insoluble complexes. Thus fecal sulfide concentrations may reflect sulfide binding capacity rather than the toxic potential of feces $[20,29]$.

The colonic bacteria ferment unabsorbed carbohydrates, producing the acetic, propionic and n-butyric acids [39]. Vogt et al. have shown that the mean percentage of butyrate absorption (30.2 $\pm 4.6 \%)$ exceeded that of acetate $(24.1 \pm 3.7 \%)(\mathrm{P}<0.05)$. The fecal molar acetate percentage was inversely correlated with the percentage of acetate absorption. There was no combination effect on short chain fatty acids absorption, and the chain-length effect suggests passive diffusion as a likely mechanism of absorption. Furthermore, fecal acetate may reflect absorption, rather than production of colonic acetate [39].

The level of ulcerations of different sections of the large intestine in rats of the first and second experimental groups under the effect of specific conditions during 25 days was studied (Table 4). Ulceration on the fifth day was not detected. However, ulcers began to form by the $10^{\text {th }}$ day. The highest level of the ulceration on the twentyfifth day in both animals groups was determined. In this case, the largest number of ulcers was found in the descending colon compared to other parts of large intestine in the rats.

Table 4. The level of ulcerations in the second and third groups of the animals under the specific conditions

\begin{tabular}{|c|c|c|c|c|c|c|c|}
\hline \multirow{2}{*}{\multicolumn{2}{|c|}{ Parameters }} & \multirow{2}{*}{ Section of the intestine } & \multicolumn{5}{|c|}{ Time of detection (days) } \\
\hline & & & Fifth & Tenth & Fifteenth & Twentieth & Twenty-fifth \\
\hline \multirow{10}{*}{ 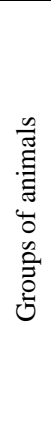 } & \multirow{5}{*}{ 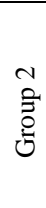 } & Caecum & nd & nd & $5 \pm 0.49$ & $9 \pm 0.88$ & $18 \pm 1.76$ \\
\hline & & Ascending colon & nd & nd & $12 \pm 1.18$ & $14 \pm 1.37$ & $24 \pm 2.35$ \\
\hline & & Transverse colon & nd & $5 \pm 0.47$ & $14 \pm 1.33$ & $17 \pm 1.62$ & $27 \pm 2.84$ \\
\hline & & Descending colon & nd & $7 \pm 0.69$ & $17 \pm 1.67$ & $20 \pm 1.96$ & $29 \pm 3.24$ \\
\hline & & Rectum & nd & $6 \pm 0.59$ & $15 \pm 1.47$ & $22 \pm 2.16$ & $26 \pm 2.75$ \\
\hline & \multirow{5}{*}{ 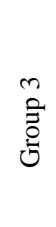 } & Caecum & nd & nd & $11 \pm 1.06^{* *}$ & $22 \pm 2.12^{* *}$ & $51 \pm 4.90^{* * *}$ \\
\hline & & Ascending colon & nd & $8 \pm 0.77$ & $27 \pm 2.62^{* *}$ & $33 \pm 3.17^{* *}$ & $68 \pm 6.54^{* * *}$ \\
\hline & & Transverse colon & nd & $13 \pm 1.25^{* *}$ & $32 \pm 3.08^{* *}$ & $48 \pm 4.62^{* * *}$ & $71 \pm 6.83^{* *}$ \\
\hline & & Descending colon & nd & $18 \pm 1.73^{* *}$ & $39 \pm 3.75^{* *}$ & $53 \pm 5.10^{* * *}$ & $78 \pm 7.50^{* * *}$ \\
\hline & & Rectum & nd & $15 \pm 1.44^{* *}$ & $34 \pm 3.27^{* *}$ & $52 \pm 4.94^{* *}$ & $76 \pm 7.21^{* * *}$ \\
\hline
\end{tabular}

Comment: The level of ulcerations was assessed visually and expressed as a percentage of number and size of ulcers per $\mathrm{cm}^{2}$ of the intestine; "nd" is not detected. Statistical significance of the values $\mathrm{M} \pm \mathrm{m}, \mathrm{n}=3 ;{ }^{* *} \mathrm{P}<0.01,{ }^{* * *} \mathrm{P}<0.001$, compared to second animals group.

The data on level of ulcerations in the descending colon are consistent to the results that hydrogen sulfide and acetate accumulation was also highest in the descending colon. Comparing the ulcers formation level in both animals groups, the higher level of ulcerations was observed in the third group than the second animals group. Perhaps, the sulfate-reducing bacteria $D$. piger Vib-7 and Desulfomicrobium sp. Rod-9 are more aggressive for large intestine and can be involved in much more intensive development of inflammatory bowel disease and ulcerative colitis.

Studies comparing the numbers of the sulfate-reducing bacteria or their products in feces from patients with ulcerative colitis and normal subjects have yielded varying results. One report found that people with ulcerative colitis had a $70 \%$ higher stool sulfide concentration, a

twofold increase in the sulfide production rate, and a fivefold increase in the sulfate-reducing bacteria [21].

Another research group found no increase in total fecal the sulfate-reducing bacteria; however, ulcerative colitis feces contained different ratios of the various species of the sulfate-reducing bacteria, and the bacteria of ulcerative colitis subjects were more active and grew better in a confined area $[2,7]$. In addition, those with colitis had a $28 \%$ increase in fecal sulfide levels and more than a doubling in sulfide production rates. Another study found no difference in the feces fecal the sulfate-reducing bacteria of ulcerative colitis subjects versus that of healthy controls [11].

It should be also noted that the microflora of the lumen distal colon (number of microorganisms, their ratio, the sustainability index and frequency of detection) on the 
twenty-fifth day was significantly changed in the second and third group which had received the dose with sulfate containing medium or the D. piger Vib-7 and Desulfomicrobium sp. Rod-9 compared to the control group.

Under these conditions, the number of Bifidobacterium and Lactobacillus genera significantly decreased, while the number of Clostridium, Escherichia, Proteus, Klebsiella, Staphylococcus genera, and the sulfatereducing bacteria were significantly increased. Perhaps, these microorganisms play a major role in the development of the inflammatory bowel diseases and ulcerative colitis.

The presence of $E$ coli in patients with ulcerative colitis was investigated, and it was reported that $E$ coli could be detected only in a small proportion of tissue samples $[40,41]$. Studies on mucosal adhesion of pathogenic bacteria in ulcerative colitis are controversial. A significantly enhanced adhesion of isolates of $E$ coli from stool specimens and rectal biopsies from ulcerative colitis patients to buccal epithelial cells was found in comparison with patients with infectious diarrhoea or normal controls [42]. The adhesive properties were similar to those of pathogenic intestinal $E$ coli, suggesting that virulent $E$ coli strains might participate in the pathogenesis of ulcerative colitis $[43,44]$. Another study reported adherence of only $E$. coli subtypes to rectal mucosa, however, no differences in adhesion could be found between ulcerative colitis patients and controls [45,46]. Using a hybridisation in situ technique, a significantly higher number of bacteria was found within the mucus layer and not adherent to the surface of the epithelium in ulcerative colitis patients compared with controls, independently from the degree of inflammation. Most likely the bacteria belong to a variety of species, considering the broad specificity of the probe used in this study [47]. To summarize, there was incomplete information and continuing controversy about the role, adherent properties, and subtypes of $E$ coli which might be important in the pathogenesis of ulcerative colitis [42].

Campieri and Gionchetti (2001) have reported that the role of bacteria in the pathogenesis was shown most convincingly in animal models [42]. A causative role for Bacteroides species in experimental ulcerative colitis was suggested. In a carrageenan guinea pig model of experimental colitis, germ free animals did not develop colitis until after monoassociation with Bacteroides vulgatus [48]. Subsequently, it was suggested that different strains of $B$. vulgatus led to considerable differences in the inflammatory response without correlation between the sources of strains and the severity of carrageenan induced lesions. In this model, pretreatment with metronidazole prevented colitis, while administration of Gram positive organisms or coliforms were not effective. These data suggest the need for an interaction between bacteria sensitive to metronidazole and dietary sulfate. More recently, the degree of caecal inflammation in HLA-B27 transgenic rats was shown to be correlated with levels of isolates on Bacteroides selective medium and increased anaerobic/aerobic and Bacteroides/aerobic ratios [49]. Indirect evidence for the interaction between luminal flora and the immune system exists from studies using animal models with disruptions in immunoregulatory molecules. It was reported that spontaneous colitis which consistently develops in knockout and transgenic murin models, does not occur when these mice are maintained in germ free conditions [50,51].
Fite and coauthors (2013) have shown that the bacteria belonging to the normal colonic microbiota were associated with the etiology of ulcerative colitis. Although several mucosal species have been implicated in the disease process, the organisms and mechanisms involved are unknown [52]. These authors have also characterized mucosal biofilm communities over time and determined the relationship of these bacteria to patient age and disease severity and duration. Multiple rectal biopsy specimens were taken from 33 patients with active ulcerative colitis over a period of 1 year. Real-time PCR was used to quantify mucosal bacteria in ulcerative colitis patients compared to 18 non inflammatory bowel disease controls, and the relationship between indicators of disease severity and bacterial colonization was evaluated by linear regression analysis. Significant differences in bacterial populations were detected between the ulcerative colitis mucosa and control group, which varied over the study period. High clinical activity indices and sigmoidoscopy scores were associated with enterobacteria, desulfovibrios, type E Clostridium perfringens, and Enterococcus faecalis, whereas the reverse was true for Clostridium butyricum, Ruminococcus albus, and Eubacterium rectale. Lactobacillus and Bifidobacterium numbers were linked with low clinical activity indices. Only E. rectale and Clostridium clostridioforme had high age dependence. These findings demonstrated that longitudinal variations in mucosal bacterial populations occur in ulcerative colitis and that bacterial community structure was related to disease severity [52].

In recent years, there are many reports about animal model to investigate drugs for inflammatory bowel diseases [24]. Acetic acid-induced damage of colonic mucosa is often used as the experimental animal model. Zheng and coauthors (2000) have investigated the use of this animal model by administering various concentrations of trinitrobenzenesulfonic acid [24].

The specific pathogenesis underlying inflammatory bowel disease is complex, and it is even more difficult to decipher the pathophysiology to explain for the similarities and differences between two of its major subtypes, Crohn's disease and ulcerative colitis. Low et al. (2013) have reported the therapeutic strategies and approaches tested on ulcerative colitis animal models. Ulcerative colitis is an idiopathic chronic relapsingremitting inflammatory disorder that affects the colon, characterized by diarrhea and rectal bleeding [23]. The molecular etiology of ulcerative colitis development is complex and involves genetic, microbial, environmental, and other unknown factors. The discussion of the underlying pathophysiology of ulcerative colitis and how observations from animal models that mimic ulcerative colitis contribute to better understanding of this disease and lead to advancement in novel treatment design was also reported by Low et al. (2013). There is a steady increase in the global incidence of ulcerative colitis. Currently, the prevalence in Europe and North America is 24.3 and 19.2 per 100,000 individuals, respectively, and 6.3 per 100,000 people in Asia and the Middle East. Most patients develop UC between the ages of 15 and 30 years, although individuals aged 50-70 years form another potential risk group. There are no significant differences in ulcerative colitis risk between sexes. The growing prevalence of this disease increases both economic and health care burdens [23]. Animal models of inflammatory bowel diseases have 
significant advantages - one can investigate not only factors concerned in pathogenesis, but also the secondary effects of ulceration, e.g., liver changes, effects on protein metabolism, electrolytic changes in the cellular and extracellular spaces, and other systemic complications [53,54].

Barnet and coauthors (2011) have reported that these animal models may also be used to study the influence of drugs or other potential therapies on the pathogenesis and course of the disease process. However, none of the current inflammatory bowel diseases models in itself constitutes a faithful equivalent for the human diseases. It may therefore be essential to evaluate the effect of any candidate therapies in several inflammatory bowel diseases models. The wide variety of models of inflammatory bowel diseases includes chemically-induced models, adoptive transfer models, and genetically modified models such as gene knockouts and transgenic animals [53].

Barnet et al. (2011) also concluded that there is clearly still a role for animal models of the inflammatory bowel diseases, however more appropriate use of new information and technologies, better classification of inflammatory bowel diseases based on both phenotypic and genetic information, and closer alliances between fundamental biological researchers and clinicians are required to ensure that the key lessons from these models are effectively moved into clinical practice. This should enable more successful strategies for the prevention and amelioration of this debilitating condition [54].

\section{Conclusions}

Based on all obtained studies results, we can conclude that injection of the sulfate containing medium or suspension with $D$. piger Vib-7 and Desulfomicrobium sp. Rod-9 in the gastrointestinal tract of the rats caused significant changes in the microflora of the animals during 25 days of the experiment. Under these conditions, structure of the microbiocenosis, number of the microorganisms, their ratio, sustainability index and frequency on the twenty-fifth day were significantly changed in both experimental groups of the animal. The number of Bifidobacterium, Lactobacillus, Peptostreptococcus and Peptococcus genera were decreased. The number of Clostridium, Escherichia, Staphylococcus, Proteus genera, and the sulfate-reducing bacteria were increased. There was a significant colonization of the lumen colon by the lactose-negative Escherichia and Klebsiella genera.

According to the results of sulfide and acetate accumulation, the highest level of these bacterial metabolites was detected in the descending colon during twenty-five days. Sulfide and acetate concentration increased directly proportional to the duration of injection of the dose of the sulfate containing medium or the suspension with the sulfate-reducing bacteria. However, significantly increased concentration of these compounds was observed in the fecal samples from the third group of the rats compared to the second group of the animals, which reserved only the sulfate containing medium. These data were consistent to results of the ulcerations in the third groups where the level of ulcer formation was also much higher than second group. Perhaps, the contribution of the D. piger Vib-7 and Desulfomicrobium sp. Rod-9 injected in the gastrointestinal tract was significantly more than endogenous intestinal microflora of the rats.

Thus, the injection of the sulfate containing medium or suspension with the sulfate-reducing bacteria in the gastrointestinal tract of the rats leads to a change in the qualitative and quantitative composition of the normal intestinal microflora (dysbiosis), which may be the result of the development of the inflammatory bowel disease, ulcerative colitis, and various pathological processes. These studies might help in predicting the development of diseases of the gastrointestinal tract, by providing further details on the etiology of bowel diseases which are very important for the clinical diagnosis of these disease types. Understanding the role of sulfate-reducing bacteria in colonic conditions would be enhanced by the ability to inhibit the number of the sulfate-reducing bacteria and/or reduce the production of sulfide and acetate. This would help to clarify the factors influencing sulfide production in the human colon.

\section{Acknowledgements}

The author expresses his gratitude to Yurij J. Beno, $\mathrm{PhD}$ student at Department of Biophysics and Bioinformatics of Biology Faculty at Ivan Franko National University of Lviv for his assistance in performing the statistical analysis.

\section{Conflict of Interest}

The author confirms that this article content has no conflicts of interest.

\section{References}

[1] Barton, L.L. and Hamilton, W.A., Sulphate-Reducing Bacteria. Environmental and Engineered Systems, Cambridge University Pres, Cambridge, 2010, 553 p.

[2] Gibson, G.R., Cummings, J.H., Macfarlane, G.T., "Growth and activities of sulphate-reducing bacteria in gut contents of health subjects and patients with ulcerative colitis”, FEMS Microbiol Ecol, 86. 103-112. 1991.

[3] Gibson, G.R., Macfarlane, G.T., Cummings, J.H., "Sulphatereducing bacteria and hydrogen metabolism in the human large intestine”, Gut, 34. 437-439. 1993

[4] Kushkevych, I.V., "Sulfate-reducing bacteria of the human intestine. I. Dissimilatory sulfate reduction”, Sci Int J Biological studies/Studia Biologica, 6(1). 149-180. 2012.

[5] Brenner, D.J., Krieg, N.R., Staley, J.T., Garrity, G.M. Bergey's manual of Systematic Bacteriology. The Proteobacteria, Part C: The Alpha-, Beta-, Delta-, and Epsilonproteobacteria. Second Edition. Printed in the United States of America, 1388 p. 2005.

[6] Postgate, J.R., The sulfate-reducing bakteria, Cambridge University Press, Cambridge, 1984, 199 p.

[7] Gibson, G.R., Macfarlane, S., Macfarlane, G.T., "Metabolic interactions involving sulphate-reducing and methanogenic bacteria in the human large intestine”, FEMS Microbiol Ecol, 12. 117-125. 1993.

[8] Kushkevych, I.V., "Sulfate-reducing bacteria of the human intestine. II. The role in the diseases development”, Sci Int J Biological studies/Studia Biologica, 6(2). 221-250. 2012.

[9] Kushkevych, I.V. and Moroz, O.M., "Growth of various strains of sulfate-reducing bacteria of human large intestine”, Sci Int J Biological studies/Studia Biologica, 6(3). 107-116. 2012.

[10] Macfarlane, S., Hopkins, M.J., Macfarlane, G.T., "Bacterial growth and metabolism on surfaces in the large intestine”, Microb Ecol Health Dis, 2. 64-72. 2000. 
[11] Levine, J., Ellis, C.J., Furne, J.K., Springfield, J. and Levitt, M.D., "Fecal Hydrogen Sulfide Production in Ulcerative Colitis", The American J Gastroenterol, 93(1). 83-87. 1998.

[12] Loubinoux, J., Bronowicji, J.-P., Pereira, I.A. et al., "Sulphatereducing bacteria in human feces and their association with inflammatory diseases”, FEMS Microbiol Ecol, 40. 107-112. 2002.

[13] Loubinoux, J., Mory, F., Pereira, I.A. et al., "Bacteremia caused by a strain of Desulfovibrio related to the provisionally named Desulfovibrio fairfieldensis”, J Clin Microbiol, 38. 931-934. 2000.

[14] Pitcher, M.C., Cummings, J.H., "Hydrogen sulphide: a bacterial toxin in ulcerative colitis?”, Gut, 39. 1-4. 1996.

[15] Pitcher, M.C.L., Beatty, E.R., Gibson, G.R., et al., "Incidence and activities of sulphate-reducing bacteria in patients with ulcerative colitis”, Gut, 36. A63 (abstract). 1995.

[16] Macfarlane, S. and Dillon, J.F., "Microbial biofilms in the human gastrointestinal tract”, J Appl Microbiol, 102. 1187-1196. 2007.

[17] Zinkevich, V.V., Beech, I.B. "Screening of sulfate-reducing bacteria in colonoscopy samples from healthy and colitic human gut mucosa”, FEMS Microbiol. Ecol, 34. 147-155. 2000.

[18] Sekirov, I., Russell, S.L., Antunes, L.C.M., Finlay, B.B., "Gut Microbiota in Health and Disease", Physiol Rev, 90. 859-904. 2010.

[19] Cummings, J.H., Macfarlane, G.T., Macfarlane, S., "Intestinal Bacteria and Ulcerative Colitis”, Curr Issues Intest Microbiol, 4. 9-20. 2003.

[20] Levitt, M.D., Furne, J., Springfield, J., Suarez, F. and Demaster, E., "Detoxification of hydrogen sulfide and methanethiol in the cecal mucosa”, J Clin Invest, 104. 1107-1114. 1999.

[21] Florin, T.H.J., Gibson, G.R., Neale, G. et al., "A role for sulfate reducing bacteria in ulcerative colitis?”, Gastroenterology, 98. A170 (abstract). 1990.

[22] Perse, M. and Cerar, A., "Dextran Sodium Sulphate Colitis Mouse Model: Traps and Tricks”, J Biomed and Biotech, Article ID 718617. 1-13. (2012).

[23] Low, D., Nguyen, D.D., Mizoguchi, E., “Animal models of ulcerative colitis and their application in drug research”, Drug Design, Development and Therapy, 7. 1341-1357. 2013.

[24] Zheng, L., Gao, Z.Q. and Wang, S.X., "A chronic ulcerative colitis model in rats", World Journal of Gastroenterology, 6(1). 150-152. 2000

[25] Nell, S., Suerbaum, S., Josenhans, C. The impact of the microbiota on the pathogenesis of IBD: lessons from mouse infection models, Nat Rev Microbiol, 8(8). 564-577. 2010.

[26] Kushkevych, I.V., "Identification of sulfate-reducing bacteria strains of human large intestine”, Sci Int $J$ Biological studies/Studia Biologica, 7(3). 115-124. 2013.

[27] Kushkevych, I.V., Bartos, M., Bartosova, L., "Sequence analysis of the 16S rRNA gene of sulfate-reducing bacteria isolated from human intestine”, Int J Curr Microbiol Appl Sci, 3(2). 239-248. 2014.

[28] Ohge, H., Furne, J.K., Springfield, J., Sueda, T., Madoff, R.D., Levitt, M.D., "The effect of antibiotics and bismuth on fecal hydrogen sulfide and sulfate-reducing bacteria in the rat", FEMS Microbiol Lett, 228. 137-142. 2003.

[29] Levitt, M.D., Springfield, J., Furne, J., Koenig, T. and Suarez, F., "Physiology of sulfide in the rat colon: use of bismuth to assess colonic sulfide production”, J Appl Physiol, 92. 1655-1660. 2002.

[30] Bailey, N.T.J. Statistical Methods in Biology, Cambridge University Press, Cambridge, 1995, 252 p.

[31] Chen, P.Y., Popovich, P.M., Correlation: Parametric and Nonparametric Measures. Sage University Papers Series on Quantitative Applications in the Social Sciences, 2002, 104 p.

[32] Beauchamp, R.O., Bus, J.S., Popp, J.A., Boreiko, C.J. and Andjelkovich, D.A., "A critical review of the literature on hydrogen sulfide toxicity”, Crit Rev Toxicol, 13. 25-97. 1984.

[33] Suarez, F.L., Furne, J., Springfield, J. and Levitt, M.D., "Production and elimination of sulfur-containing gases in the rat colon”, Am J Physiol, 274. G727-G733. 1998.
[34] Chapman, M.A.S., Grahn, M.F., Boyle, M.A. et al., "Butyrate oxidation is impaired in the colonic mucosa of sufferers of quiescent ulcerative colitis”, Gut, 35. 73-76. 1994.

[35] Roediger, W.E.W., Duncan, A., Kapaniris, O. and Millard, S. "Reducing sulfur compounds of colon impair colonocyte nutrition: implications for ulcerative colitis", Gastroenterology, 104. 802809. 1993.

[36] Roediger, W.E.W., Duncan, A., Kapaniris, O., et al., "Sulphide impairment of substrate oxidation in rat colonocytes: A biochemical basis for ulcerative colitis?”, Clin Sci, 85. 623-627. 1993.

[37] Roediger, W.E.W., Nance, S., "Metabolic induction of experimental ulcerative colitis by inhibition of fatty acid oxidation”, Br J Exp Pathol, 67. 773-782. 1986.

[38] Sax, N.I., Dangerous properties of industrial materials. Van Nostrand Reinhold Company, New York, 1984.

[39] Vogt, J.A. and Wolever, T.M.S., "Fecal Acetate Is Inversely Related to Acetate Absorption from the Human Rectum and Distal Colon”, J Nutr, 133(10). 3145-3148. 2003.

[40] Walmsey, R.S., Anthony, A., Slim, R., et al., "Absence of Escherichia coli, Listeria moncytogenes, and Klebsiella pneumonae antigens within inflammatory bowel disease tissues", J Clin Pathol, 51. 657-661. 1998.

[41] Kallinowski, F., Wassmer, A., Hofmann, M.A., et al., "Prevalence of enteropathogenic bacteria in surgically treated chronic inflammatory bowel disease", Hepatogastroenterology, 45. 15521558. 1998.

[42] Campieri, M., and Gionchetti, P., "Bacteria as the cause of ulcerative colitis", Gut, 48. 132-135. 2001.

[43] Burke, D.A., Axon, A.T.R., "Adhesive Escherichia coli in inflammatory bowel disease and infective diarrhoea", BMJ, 297. 102-104. 1988.

[44] Burke, D.A., Axon, A.T.R., "Ulcerative colitis and Escherichia coli with adhesive properties", J Clin Pathol, 40. 782-786. 1987.

[45] Schultsz, C., Moussa, M., van Ketel, R., et al., "Frequency of pathogenic and enteroadherent Escherichia coli in patients with inflammatory bowel disease and controls”, J Clin Pathol, 50. 573579. 1997.

[46] Von Wulffen, H, Rüssmann, H., Karch, H., et al., "VerocytoxinProducing Escherichia coli O2:H5 isolated from patients with ulcerative colitis", Lancet; 1. 1449-1450. 1989.

[47] Schultsz, C., van den Berg, F.M., Ten Kate F.W. et al., "The intestinal mucus layer from patients with inflammatory bowel disease harbours numbers of bacteria compared with controls", Gastroenterology, 117. 1089-1097. 1999.

[48] Onderdonk, A.B., Bronson, R., Cisneros, R., "Comparison of bacteroides vulgatus strains in the enhancement of experimental ulcerative colitis”, Infect Immun, 55. 835-836. 1987.

[49] Rath, H.C., Ikeda, J.S., Linde. H.-J., et al., "Varying caecal bacterial loads influences colitis and gastritis in HLA-B27 transgenic rats”, Gastroenterology, 116. 310-319. 1999.

[50] Ehrhardt, R.O., Lúdvíksson, B.R., Gray, B., et al., "Induction and prevention of colonic inflammation in IL-2-deficient mice”, $J$ Immunology, 158. 566-573. 1997.

[51] Kuhn, R., Lohler, J., Rennick, D., et al., Interleukin-10-deficient mice develop chronic enterocolitis. Cell, 75. 263-274. 1993.

[52] Fite, A., Macfarlane, S., Furrie, E. et al., "Longitudinal Analyses of Gut Mucosal Microbiotas in Ulcerative Colitis in Relation to Patient Age and Disease Severity and Duration”, J Clin Microbiol, 51(3). 849-856. 2013.

[53] Rowan, F.E., Docherty, N.G., Coffey, J.C. et al., "Sulphatereducing bacteria and hydrogen sulphide in the aetiology of ulcerative colitis”, British J Surgery, 96. 151-158. 2009.

[54] Barnett, M. and Fraser, A. "Animal Models of Colitis: Lessons Learned, and Their Relevance to the Clinic", Ulcerative Colitis, 161-178. 2011. 